\title{
A Review on Bladder Tumor Antigens
}

\author{
Obeagu Emmanuel Ifeanyi* \\ Department of University Health Services, Michael Okpara University of Agriculture, Nigeria
}

Submission: January 23, 2018; Published: February 12, 2018

"Correspondence Address: Obeagu Emmanuel Ifeanyi, Diagnostic Laboratory Unit, Department of University Health Services, Michael Okpara University of Agriculture, Umudike, Abia State, Nigeria, Email: emmanuelobeagu@yahoo.com

Abstract

The bladder is a hollow organ in the pelvis with flexible, muscular walls. Its main function is to store urine before it leaves the body. Urine is made by the kidneys and is then carried to the bladder through tubes called ureters. The bladder is an organ located in the lower abdominal area near the pelvic bones that acts as a reservoir for urine. Almost all bladder cancers originate in the urothelium, which is a 3-to 7-cell mucosal layer within the muscular bladder. Squamous cell carcinoma of the bladder can involve multiple sites; however, the lateral wall and trigone are more commonly involved by this tumor. All small cell carcinomas of the urinary system identified so far have been located in the urinary bladder, most commonly in the dome and vesical lateral wall. Bladder cancer begins when cells in the urinary bladder start to grow uncontrollably. As more cancer cells develop, they can form a tumor and spread to other areas of the body. Normally, cells in the body will grow and divide to replace old or damaged cells in the body. This growth is highly regulated, and once enough cells are produced to replace the old ones, normal cells stop dividing. Tumors occur when there is an error in this regulation and cells continue to grow in an uncontrolled way. Tumors can either be benign or malignant. Cytological examination of urine (looking for abnormal cells in urine) has been the most commonly tested screening tool. Routine urinalysis, performed as part of normal health maintenance, will detect any presence of blood in the urine.

\section{Anatomy of the Bladder}

The bladder is a hollow organ in the pelvis with flexible, muscular walls. Its main function is to store urine before it leaves the body. Urine is made by the kidneys and is then carried to the bladder through tubes called ureters. The bladder is an organ located in the lower abdominal area near the pelvic bones that acts as a reservoir for urine. The bladder expands and can hold about half of a liter of urine, but a person usually feels the urge to urinate when the bladder is $25 \%$ full when you urinate, the muscles in the bladder contract, and urine is forced out of the bladder through a tube called the urethra [1]. The wall of the bladder has several layers, which are made up of different types of cells.

In males, the seminal vesicles, vas deferens, ureters, and rectum border the inferoposterior aspect of the bladder. Anterior to the bladder is the space of Retzius, which is composed of fibroadipose tissue and the prevesical fascia. The dome and posterior surface of the bladder are covered by parietal in females; the posterior peritoneal reflection is continuous with the uterus and vagina. The vascular supply to the bladder arrives primarily via the internal iliac (hypogastric) arteries, branching into the superior, middle, and inferior vesical arteries, which are often recognizable as lateral and posterior pedicles. The arterial supply also arrives via the obturator and inferior gluteal artery and, in females, via the uterine and vaginal arteries. Bladder venous drainage is a rich network that often parallels the named arterial vessels, most of which ultimately drain into the internal iliac vein.

Initial lymphatic drainage from the bladder is primarily into the external iliac, obturator, internal iliac (hypogastric), and common iliac nodes. Following the drainage to these sentinel pelvic regions, spread may continue to the presacral, paracaval, interaortocaval, and para-aortic lymph node chains. Almost all bladder cancers originate in the urothelium, which is a 3- to 7 -cell mucosal layer within the muscular bladder. Squamous cell carcinoma of the bladder can involve multiple sites; however, the lateral wall and trigone are more commonly involved by this tumor. All small cell carcinomas of the urinary system identified so far have been located in the urinary bladder, most commonly in the dome and vesical lateral wall.

Most bladder cancers start in the innermost lining of the bladder, which is called the urothelium or transitional epithelium. As the cancer grows into or through the other layers in the bladder wall, it becomes more advanced and can be harder to treat. Over time, the cancer might grow outside the bladder and into nearby structures. It might spread to nearby lymph nodes, or to other parts of the body (if bladder cancer spreads, it often goes first to distant lymph nodes, the bones, the lungs, or the liver) [2].

The bladder wall consists of 4 main layers of tissues. The innermost lining is called the urothelium, or transitional 
epithelium, and is made up of cells known as urothelial or transitional cells. Beneath this layer is a thin layer called the lamina propria, which is made up of connective tissue, blood vessels and nerves? The next layer is called the muscularis propria, which is made of muscle. The last layer is a layer of fatty connective tissue that separates the bladder from other surrounding organs [3].

\section{Bladder Cancer}

Bladder cancer begins when cells in the urinary bladder start to grow uncontrollably. As more cancer cells develop, they can form a tumor and spread to other areas of the body. Normally, cells in the body will grow and divide to replace old or damaged cells in the body. This growth is highly regulated, and once enough cells are produced to replace the old ones, normal cells stop dividing. Tumors occur when there is an error in this regulation and cells continue to grow in an uncontrolled way. Tumors can either be benign or malignant. Although benign tumors may grow in an uncontrolled fashion sometimes, they do not spread beyond the part of the body where they started (metastasize) and do not invade into surrounding tissues. Malignant tumors, however, will grow in such a way that they invade and damage other tissues around them. They also may spread to other parts of the body, usually through the blood stream or through the lymphatic system where the lymph nodes are located [4].

Over time, the cells within a malignant tumor become more abnormal and appear less like normal cells. This change in the appearance of cancer cells is called the tumor grade, and cancer cells are described as being well- differentiated, moderatelydifferentiated, poorly- differentiated, or undifferentiated. Welldifferentiated cells are quite normal appearing and resemble the normal cells from which they originated. Undifferentiated cells are cells that have become so abnormal that often we cannot tell what types of cells they started from [5].

Cancers are described by the types of cells from which they arise. Bladder cancers arise almost exclusively from the innermost lining of the bladder, so they are referred to as transitional cell or urothelial cancer. In the United States, more than 9 out of 10 bladder cancers are called transitional cell carcinomas. This simply means that the cancer started in the lining of the bladder, which is made up of transitional cells that appear elliptical under the microscope. Less commonly, other types of cancers can arise from the lining of the bladder, called adenocarcinomas, Squamous cell carcinomas and small cell carcinomas.

Commonly, bladder cancers grow in a "papillary" growth pattern. When a bladder cancer grows this way, it can be invasive (invading into tissues) or noninvasive (not invading into tissues at all), and hence not having a risk for distant spread, as long as it is treated. In addition, there can be precancerous lesions in the bladder, called carcinoma-in- situ. Carcinoma-in-situ occurs when the lining of the bladder undergoes changes similar to cancerous changes without any invasion into the deeper tissues. Hence, while the cells themselves have cancer-like qualities, no invasion has occurred. However, both papillary bladder cancers and cancer-in-situ may become invasive, so treatment is very important [6].

\section{Types of Bladder Cancer}

\section{Urothelial Carcinoma (transitional cell carcinoma)}

Urothelial carcinoma, also known as transitional cell carcinoma (TCC), is by far the most common type of bladder cancer. In fact, if you are told you have bladder cancer it is almost certain to be an urothelial carcinoma [7]. These cancers start in the urothelial cells that line the inside of the bladder. Urothelial cells also line other parts of the urinary tract, such as the part of the kidney that connects to the ureter (called the renal pelvis), the ureters, and the urethra. Patients with bladder cancer sometimes have other tumors in these places, so the entire urinary tract needs to be checked for tumors. In North America, South America, Europe, and Asia, the most common type of urothelial tumor diagnosed is transitional (urothelial) cell carcinoma (TCC); it constitutes more than $90 \%$ of bladder cancers in those regions. TCC can arise anywhere in the urinary tract, including the renal pelvis, ureter, bladder, and urethra, but it is usually found in the urinary bladder.

\section{Squamous Cell Carcinoma}

Squamous cells appear in your bladder in response to infection and irritation. Over time they can become cancerous. Squamous cell bladder cancer is rare in the United States. It's more common in parts of the world where a certain parasitic infection (schistosomiasis) is a prevalent cause of bladder infections. Under a microscope, the cells look much like the flat cells that are found on the surface of the skin. Nearly all squamous cell carcinomas are invasive. Squamous cell carcinoma (SCC) is the second most common cell type associated with bladder cancer in industrialized countries. In the United States, around 5\% of bladder cancers are SCCs. Worldwide, however, SCC is the most common form of bladder cancer, accounting for $75 \%$ of cases in developing nations. In the United States, the development of SCC is associated with persistent inflammation from long-term indwelling Foley catheters and bladder stones, as well as, possibly, infections. In developing nations, SCC is often associated with bladder infection by Schistosoma haematobium.

\section{Adenocarcinoma}

Adenocarcinoma begins in cells that make up mucussecreting glands in the bladder. Adenocarcinoma of the bladder is rare in the United States. Some bladder cancers include more than one type of cell. Only about $1 \%$ of bladder cancers are adenocarcinomas. The cancer cells have a lot in common with gland-forming cells of colon cancers. Nearly all adenocarcinomas of the bladder are invasive. A bladder cancer can also be 
described as superficial or non-muscle invasive. These terms include both non- invasive tumors as well as any invasive tumors that have not grown into the main muscle layer of the bladder.

\section{Invasive versus non-invasive bladder cancer}

Bladder cancers are often described based on how far they have invaded into the wall of the bladder:

Non-invasive cancers are still in the inner layer of cells (the transitional epithelium) but have not grown into the deeper layers. Invasive cancers have grown into deeper layers of the bladder wall. These cancers are more likely to spread and are harder to treat.

\section{Papillary versus flat cancer}

Bladder cancers are also divided into 2 subtypes, papillary and flat, based on how they grow.

\section{Papillary Carcinomas}

Papillary carcinomas grow in slender, finger-like projections from the inner surface of the bladder toward the hollow center. Papillary tumors often grow toward the center of the bladder without growing into the deeper bladder layers. These tumors are called non-invasive papillary cancers. Very low-grade (slow growing), non-invasive papillary cancer is sometimes called papillary urothelial neoplasm of low-malignant potential (PUNLMP) and tends to have a very good outcome [8].

\section{Flat Carcinomas}

Flat carcinomas do not grow toward the hollow part of the bladder at all. If a flat tumor is only in the inner layer of bladder cells, it is known as a non-invasive flat carcinoma or a flat carcinoma in situ (CIS). If either a papillary or flat tumor grows into deeper layers of the bladder, it is called an invasive urothelial (or transitional cell) carcinoma. Small cell carcinoma: Less than $1 \%$ of bladder cancers are small-cell carcinomas, which start in nerve-like cells called neuroendocrine cells. These cancers often grow quickly and typically need to be treated with chemotherapy like that used for small cell carcinoma of the lung. Sarcoma: Sarcomas start in the muscle cells of the bladder, but they are rare. More information about sarcomas can be found in Sarcoma - Adult Soft Tissue Cancer and Rhabdomyosarcoma. These less common types of bladder cancer (other than sarcoma) are treated similar to TCCs, especially for early stage tumors, but if chemotherapy is needed, different drugs might be use.

\section{Causes of Bladder Cancer}

Up to $80 \%$ of bladder cancer cases are associated with environmental exposure and a number of occupations involve exposure to substances that may increase risk for bladder cancer. Of occupationally related bladder cancer cases, the incidence rate is highest in workers Tobacco use is by far the most common cause of bladder cancer in the World, and is increasing in importance in some developing countries. Smoking duration and intensity are directly related to increased risk. It is estimated that in 2015 in the United States there will be 74,000 new cases of bladder cancer, resulting in 16,000 deaths [9].

It is the sixth most common cancer diagnosis in the United States, being the third most common in men and eleventh most common in women. In the US, bladder cancer tends to affect older men more frequently; with men affected more than women by a 3:1 ratio and $2 / 3$ of the cases diagnosed in people over the age of 65 . Cigarette smoking is the largest risk factor for bladder cancer. It is estimated that about half of bladder cancers are caused by cigarette smoking. The risk of being diagnosed with bladder cancer is increased two to four times if you are a smoker [10]. Other risk factors for developing bladder cancer include, family history, genetic mutations, occupational exposure to chemicals (especially those processed in; Paint, Dye Metal and petroleum products Previous cancer treatment with cyclophosphamide ifosfamide, Pelvic radiation Exposure to arsenic especially in well water aristolochic (a Chinese herb) Beauticians Dry cleaners, Painters Paper production workers, Rope-and-twine industry workers, Dental workers, Physicians Barbers.

People living in urban areas are also more likely to develop bladder cancer. The etiology in these cases is thought to be multifactorial, potentially involving exposure to numerous carcinogens. Bladder infections caused by schistosoma haematobium, and neurogenic bladder and the overuse of indwelling catheters.

\section{Prevention of Bladder Cancer}

There is no sure way to prevent bladder cancer, but some risk factors have been identified, which may increase your risk of developing the condition, but there are things one can do that could lower the risk.

\section{Smoking Cessation}

Smoking cessation is the best way to prevent bladder cancer. Additionally, reducing the exposure to cancer causing agents should decrease the risk of developing bladder cancer. Other than these preventative measures, decreasing the risk of invasive bladder cancer relies on early detection of symptoms and possibly screening high-risk individuals [11].

\section{Workplace safety}

Ones risk of bladder cancer could be increased if your job involves exposure to certain chemicals. Occupations linked to an increased risk of bladder cancer are manufacturing jobs involving: rubber, dyes, textiles, plastic, leather tanning and diesel fumes.

\section{Diet}

There's some evidence to suggest that a diet high in fruit and vegetables and low in fat can help to prevent bladder cancer. Even though this evidence is limited, it's a good idea to follow this type of healthy diet, as it can help to prevent other types of 
cancer, such as bowel cancer, as well as serious health conditions, including high blood pressure (hypertension), stroke and heart disease. A low-fat, high-fiber diet is recommended, including plenty of fresh fruit and vegetables (five portions a day) and whole grains. Limit the amount of salt you eat to no more than $6 \mathrm{~g}(0.2 \mathrm{oz})$ a day (one teaspoon) because too much can increase your blood pressure [12].

You should try to avoid foods that contain saturated fat, as it can increase your cholesterol levels. Foods high in saturated fat include: meat pies sausages and fatty cuts of meat, butter ghee (a type of butter often used in Indian cooking), lard, cream, cakes and biscuits, foods that contain coconut or palm oil. However, a balanced diet should include a small amount of unsaturated fat, because this will actually help to control your cholesterol levels. Comprehensive bladder cancer control involves prevention, early detection, diagnosis and treatment, rehabilitation and palliative care.

\section{Signs and Symptoms of Bladder Cancer}

The most common sign of bladder cancer is the presence of blood in the urine, called haematuria. The blood in the urine can either be noticeable by the naked eye (called gross haematuria), or noted only when the urine is analyzed in a laboratory (called microscopic haematuria). Other signs of bladder cancer could include increased frequency of urination, a feeling of urgency to urinate, nocturia (waking up at night due to having to urinate), pain (burning) with urination, and the feeling of incomplete bladder emptying. These are all caused by irritation of the bladder wall by the tumor. In advanced cases of bladder cancer, the tumor can actually obstruct either the entrance of urine into the bladder or the exit of urine from the bladder. This causes severe flank pain, infection, and damage to the kidneys.

\section{Screening tests used for bladder cancer}

There are no standard screening tests used for bladder cancer in people who have not had bladder cancer.

Cystoscopy and urine cytology may be used in people with a history of bladder cancer.

\section{Bladder Cancer Diagnosis}

Cytological examination of urine (looking for abnormal cells in urine) has been the most commonly tested screening tool. It involves testing urine for the presence of abnormal cells, which would indicate the possibility of a cancer [13]. This method is fairly inexpensive and without risk to the patient. However, a fair amount of cancers can be missed using this method. Also, the incidence of preclinical (too small to cause any symptoms) bladder cancer in the general population is likely too low for cytologic examination of urine to be useful as a mass screening tool [14]. Routine urinalysis, performed as part of normal health maintenance, will detect any presence of blood in the urine. If blood is detected and is not due to another cause (such as infection), further tests should be carried out.
Anyone with either gross or microscopic hematuria should undergo a work-up to ensure the symptoms are not from bladder (or other) cancer. Often, the first thing that is done is a urine cytology, which as mentioned above, is looking at the urine under a microscope to detect cancerous appearing cells. Again, if these cells are seen, a diagnosis of cancer may be made. However, the test does not detect all cases of bladder cancer. X-ray imaging of the upper urinary tract (including the ureters and kidneys) may be performed to diagnose bladder cancer, or after a diagnosis of bladder cancer to rule out involvement of these structures with the cancer. Ultrasound can be used to study the kidneys, and a CT scan is often very good at studying the entire length of the urinary tract. One of the traditional methods of studying the (upper) urinary tract is with an intravenous pyelogram (IVP) [15].

This involves administering a dye through a patient's vein and taking a regular $\mathrm{x}$-ray a short time later. The dye is excreted via the kidneys and urine and can be seen on the $\mathrm{x}$-ray, showing the full extent of the kidney collecting system, ureters, and often the bladder. Though the above tests are useful, the mainstay of diagnosis and staging is endoscopic evaluation with cystoscopy. This involves placing a fiberoptic camera into the bladder via the urethra. Cystoscopy allows for direct visualization of the entire bladder and also allows for biopsy of any suspicious lesions. If the biopsy reveals cancer, a repeat cystoscopy and resection (called a transurethral resection (TUR)) is done to completely evaluate the tumor and the extent and depth of disease [16]. When a diagnosis of bladder cancer is made, a complete physical examination is done as well as the previously mentioned radiologic studies to fully evaluate the urinary tract, the local extent of disease, and any metastatic disease.

\section{Pathogenesis}

The pathogenesis of bladder cancer is being analyzed at several levels of biological organization, i.e., population groups, individual whole animal, tissue, cell, molecule, etc. Each of these levels provides opportunities for mechanistic studies. Yet the integration of these several levels into a cohesive fabric is incomplete. From a clinical point of view, the following seem of importance to human bladder cancer pathogenesis. The initiation, promotion, and progression of bladder cancer involve several factors acting concurrently or sequentially. These factors appear to be naturally occurring or synthetically created chemicals present in the external environment. Human exposures to these agents may begin in utero, and varying, dynamic qualitative and quantitative exposure patterns continue through developmental and adult life [17].

Apparent latent periods of development of clinical bladder cancer may be as short as one, or as long as 50 years or more. Individuals may exhibit differential susceptibility to vesical carcinogens, perhaps through phenotypic differences in quantitative biotransformation routes. Differences in bladder epithelial cell susceptibilities probably also occur, as well as 


\section{Cancer Therapy \& Oncology International Journal}

varying local tissue and generalized resistance to neoplasia formation. Older individuals do not appear to be more resistant to bladder carcinogenesis [18]. A number of animal model systems have been developed for the study of the in vivo, cellular, and molecular pathogenesis of bladder cancer. These models replicate many of the known salient features of human bladder cancer. Through use of appropriate whole animal models in conjunction with investigations of human and animal bladder cells and tissues in culture, controlled mechanistic and quantitative studies of bladder cancer pathogenesis should rapidly develop.

\section{Genetic factors in Pathogenesis}

Divergent, yet interconnected and overlapping, molecular pathways are likely responsible for the development of noninvasive and invasive bladder tumors. Somatic mutations in fibroblast growth receptor3 (FGFR-3) and tumor protein p53 (TP53) in tumor cells appear to be important early molecular events in the noninvasive and invasive pathways, respectively. FGFR-3, Ras, and PIK3CA mutations occur with high frequency in noninvasive tumors, leading to up regulation of Akt and mitogen-activated protein kinase (MAPK). Loss of heterozygosity $(\mathrm{LOH})$ on chromosome 9 is among the most frequent genetic alterations in bladder tumors and is considered an early event. Large numbers of genomic changes have been detected using karyotyping and comparative genomic hybridization (CGH) analysis in urothelial carcinoma. Numerically common are losses of $2 q, 5 q, 8 p, 9 p, 10 q, 18 q$, and Y. Gains of 1q, 5p, 8q, and 17q are frequently present, and high-level amplifications can be found; however, the target genes in the regions of amplifications have not been conclusively identified. Alterations in the TP53 gene are noted in approximately $60 \%$ of invasive bladder cancers. Progression-free survival is significantly shorter in patients with TP53 mutations and is an independent predictor of death among patients with muscle-invasive bladder cancer.

Additionally, alterations in retinoblastoma (Rb), PTEN, and p16 are common in high-grade invasive cancers. Over expression of JUN, MAP2K6, STAT3, and ICAM1 and molecules associated with survival (Bcl-2, caspase-3, p53, survivin), as well as insensitivity to antigrowth signals (p53, p21, p16, pRB), has been demonstrated. In advanced disease, multiple mechanisms may lead to tumor progression. These include those that promote proliferation, survival, invasion, and metastasis, as well as those that involve deficiencies in DNA damage repair and the finding of stem like cells.

In addition to tumor cell alterations, the microenvironment may promote tumor growth by paracrine influences, including vascular endothelial growth factor (VEGF) production and aberrant E-cadherin expression. Finally, a growing body of research over the last decade indicates that epigenetic alterations may silence tumor suppressor genes and that they represent important events in tumor progression.

\section{Epidemiology}

\section{Occurrence in the United States}

The American Cancer Society estimates that 76,960 new cases of bladder cancer will be diagnosed in the United States in 2016 and that 16,390 people will die of the disease. The incidence of bladder cancer increases with age, with the median age at diagnosis being 65 years; bladder cancer is rarely diagnosed before age 40 years. Bladder cancer is about 3 times more common in men than in women. Over the past 2 decades, however, the rate of bladder cancer has been stable in men but has increased in women by $0.2 \%$ annually. The male predominance in bladder cancer in the United States reflects the prevalence of transitional cell carcinoma (TCC). With SCC in contrast to TCC the male-to-female incidence ratio is $1: 2$.

Bladder cancer is the fourth most common cancer in men in the United States, after prostate, lung, and colorectal cancer, but it is not among the top 10 cancers in women. Accordingly, more men than women are expected to die of bladder cancer in 2016, with 11,820 deaths in men versus 4570 in women. Nevertheless, women generally have a worse prognosis than men. The incidence of bladder cancer is twice as high in white men as in black men in the United States. However, blacks have a worse prognosis than whites.

\section{Occurrence in the United Kingdom}

Bladder cancer is the seventh most common cancer in the UK (around 10,400 people were diagnosed with the disease in 2011), and it is the seventh most common cause of cancer death (around 5,200 people died in 2012). Limited data indicate that small cell carcinoma of the urinary bladder probably has the same epidemiologic characteristics as urothelial carcinoma. Patients are more likely to be male and older than 50 years.

International occurrence-Worldwide, bladder cancer is diagnosed in approximately 275,000 people each year, and about 108,000 die of this disease. In industrialized countries, $90 \%$ of bladder cancers are TCC. In developing countries particularly in the Middle East and Africa the majority of bladder cancers are SCCs, and most of these cancers are secondary to Schistosoma haematobium infection. Recent studies report that urothelial carcinoma is the most common urologic cancer in China [19].

\section{Occurance in Africa}

In Africa, the highest incidence of SCC has been seen in schistosomal-endemic areas, notably Sudan and Egypt, where SCC ranges from two thirds to three quarters of all malignant tumors of the bladder. In recent years, a few studies from Egypt have shown a reversal of this trend due to the better control of schistosomiasis in the region, whereas in other parts of Africa the association is unchanged. Increased smoking incidence is believed to have contributed to the shift in Egypt toward TCC, which has a stronger smoking association. 


\section{Cancer Therapy \& Oncology International Journal}

\section{Phenotypes}

Clinical and pathologic data indicate that at least 3 different phenotypes, as follows, exist in urothelial carcinoma. Lowgrade proliferative lesions that develop into nonmuscle-invasive tumors; this account for approximately $80 \%$ of bladder cancers. Highly proliferative invasive tumors with a propensity to metastasize.

CIS, which can penetrate the lamina propria and eventually progress.

\section{Pathophysiology}

Bladder cancer is often described as a polyclonal field change defect with frequent recurrences due to a heightened potential for malignant transformation. However, bladder cancer has also been described as resulting from implantation of malignant cells that have migrated from a previously affected site. The latter occurs less often and may account for only a small percentage of cases. Use of the common term superficial bladder cancer should be discouraged. The term implies a harmless nature, which is misleading in many instances. Because it was used to describe the disparate disorders of low-grade papillary bladder cancer and the markedly more aggressive form, carcinoma in situ (CIS), the World Health Organization (WHO) has recommended it be abandoned [20].

In its place, the term nonmuscle-invasive bladder cancer should be used and qualified with the appropriate American Joint Committee on Cancer stage (ie, Ta, T1, Tis). Stage T1 cancer invades lamina propria but not the muscle of the bladder. Highgrade T1 tumor associated with CIS carries a relatively high risk for disease recurrence and progression (approximately 60\%). The WHO classifies bladder cancers as low grade (grades 1 and 2) or high grade (grade 3).Tumors are also classified by growth patterns: papillary (70\%), sessile or mixed (20\%), and nodular (10\%). Papillary bladder tumors such as this one are typically of low stage and grade (Ta-G1). Sessile lesions as shown usually invade muscle, although occasionally a tumor is detected at the T1-G3 stage prior to muscle invasion.

\section{Risk Factors Associated With Bladder Tumor}

A risk factor is anything that changes your chance of getting a disease such as cancer. Different cancers have different risk factors. Some risk factors, like smoking, can be changed. Others, like a person's age or family history, cannot be changed. But having a risk factor, or even several, does not mean that you will get the disease. Many people with risk factors never get bladder cancer, while others with this disease may have few or no known risk factors.

Still, it's important to know about the risk factors for bladder cancer because there may be things you can do that might lower your risk of getting it. If you are at higher risk because of certain factors, you might be helped by tests that could find it early, when treatment is most likely to be effective. Several risk factors make a person more likely to develop bladder cancer [19].

\section{Risk Factors You Can Change}

Smoking: Smoking is the most important risk factor for bladder cancer. The carcinogens in cigarettes contribute heavily towards bladder cancer. Smoking causes about half of all bladder cancers in both men and women, it is estimated that is just over a third of all cases of bladder cancer are caused by smoking. People who smoke may be up to four times more likely to develop bladder cancer than non-smokers.

Workplace exposures; certain industrial chemicals have been linked with bladder cancer. Chemicals called aromatic amines, such as benzidine and beta- naphthylamine, which are sometimes used in the dye industry, can cause bladder cancer. Workers in other industries that use certain organic chemicals also may have a higher risk of bladder cancer. Industries carrying higher risks include makers of rubber, leather, textiles, and paint products as well as printing companies. Other workers with an increased risk of developing bladder cancer include painters, machinists, printers, hairdressers (probably because of heavy exposure to hair dyes), and truck drivers (likely because of exposure to diesel fumes).

Drugs; Certain medicines or herbal supplements According to the US Food and Drug Administration (FDA), use of the diabetes medicine pioglitazone (Actos) for more than one year may be linked with an increased risk of bladder cancer. Dietary supplements containing aristolochic acid (mainly in herbs from the Aristolochia family) have been linked with an increased risk of urothelial cancers, including bladder cancer.

Genetics and family history; People who have family members with bladder cancer have a higher risk of getting it themselves. Sometimes this may be because the family members are exposed to the same cancer-causing chemicals (such as those in tobacco smoke). They may also share changes in some genes (like GST and NAT) that make it hard for their bodies to break down certain toxins, which can make them more likely to get bladder cancer. A small number of people inherit a gene syndrome that increases their risk for bladder cancer. For example: A mutation of the retinoblastoma (RB1) gene can cause cancer of the eye in infants, and also increases the risk of bladder cancer. Cowden disease, caused by mutations in the PTEN gene, is linked mainly to cancers of the breast and thyroid. People with this disease also have a higher risk of bladder cancer. Lynch syndrome (also known as hereditary non-polyposis colorectal cancer, or HNPCC) is linked mainly to colon and endometrial cancer. People with this syndrome might also have an increased risk of bladder cancer (as well as other cancers of the urinary tract).

Arsenic in drinking water; Arsenic in drinking water has been linked with a higher risk of bladder cancer in some parts of the world. The chance of being exposed to arsenic depends 


\section{Cancer Therapy \& Oncology International Journal}

on where you live and whether you get your water from a well or from a public water system that meets the standards for low arsenic content. For most Americans, drinking water is not a major source of arsenic. Not drinking enough fluids; People who drink a lot of fluids, especially water, each day tend to have lower rates of bladder cancer. This might be because they empty their bladders more often, which could keep chemicals from lingering in their bladder.

\section{Risk Factors You Cannot Change}

Race and ethnicity: Whites are about twice as likely to develop bladder cancer as African Americans and Hispanics. Asian Americans and American Indians have slightly lower rates of bladder cancer. The reasons for these differences are not well understood.

i. Age: The risk of bladder cancer increases with age. About 9 out of 10 people with bladder cancer are older than 55 .

ii. Gender: Bladder cancer is much more common in men than in women. Personal history of bladder or other urothelial cancer: Urothelial carcinomas can sometimes form in different areas in the bladder, as well as in the lining of the kidney, the ureters, and urethra.

Having a cancer in the lining of any part of the urinary tract puts you at higher risk of having another cancer, either in the same area as before, or in another part of the urinary tract. This is true even when the first tumor is removed completely. For this reason, people who have had bladder cancer need careful follow-up to look for new cancers.

iii. Bladder birth Defects: Before birth, there is a connection between the belly button and the bladder. This is called the urachus. If part of this connection remains after birth, it could become cancerous. Cancers that start in the urachus are usually adenocarcinomas, which are made up of cancerous gland cells. About one-third of the adenocarcinomas of the bladder start here.

iv. Radiotherapy: Radiotherapy to treat previous cancers near the bladder, such as bowel cancer previous treatment with certain chemotherapy medications, such as cyclophosphamide and cisplatin, previous surgery to remove part of the prostate gland, during treatment for benign prostate enlargement having diabetes bladder cancer is thought to be linked to certain treatments for type 2 diabetes [21].

Bladder cancer is any of several types of cancer arising from the epithelial lining (i.e., the urothelium) of the urinary bladder. Rarely the bladder is involved by non-epithelial cancers, such as lymphoma or sarcoma, but these are not ordinarily included in the colloquial term "bladder cancer." It is a disease in which abnormal cells multiply without control in the bladder. The most common type of bladder cancer recapitulates the normal histology of the urothelium and is known as transitional cell carcinoma or more properly urothelial cell carcinoma. Fiveyear survival rates in the United States are around $77 \%$. Bladder cancer is the 9th leading cause of cancer with 430,000 new cases and 165,000 deaths occurring in 2012 [22].

\section{Genetic factors in Pathogenesis}

Divergent, yet interconnected and overlapping, molecular pathways are likely responsible for the development of noninvasive and invasive bladder tumors. Somatic mutations in fibroblast growth receptor3 (FGFR-3) and tumor protein p53 (TP53) in tumor cells appear to be important early molecular events in the noninvasive and invasive pathways, respectively. FGFR-3, Ras, and PIK3CA mutations occur with high frequency in noninvasive tumors, leading to up regulation of Akt and mitogenactivated protein kinase (MAPK). Loss of heterozygosity (LOH) on chromosome 9 is among the most frequent genetic alterations in bladder tumors and is considered an early event [23].

Large numbers of genomic changes have been detected using karyotyping and comparative genomic hybridization (CGH) analysis in urothelial carcinoma. Numerically common are losses of 2q, 5q, 8p, 9p, 10q, 18q, and Y. Gains of 1q, 5p, 8q, and 17q are frequently present, and high-level amplifications can be found; however, the target genes in the regions of amplifications have not been conclusively identified. Alterations in the TP53 gene are noted in approximately $60 \%$ of invasive bladder cancers. Progression-free survival is significantly shorter in patients with TP53 mutations and is an independent predictor of death among patients with muscle-invasive bladder cancer. Additionally, alterations in retinoblastoma (Rb), PTEN, and p16 are common in high-grade invasive cancers. Over expression of JUN, MAP2K6, STAT3, and ICAM1 and molecules associated with survival (Bcl2 , caspase-3, p53, survivin), as well as insensitivity to antigrowth signals (p53, p21, p16, pRB), has been demonstrated.

In advanced disease, multiple mechanisms may lead to tumor progression. These include those that promote proliferation, survival, invasion, and metastasis, as well as those that involve deficiencies in DNA damage repair and the finding of stem like cells. In addition to tumor cell alterations, the microenvironment may promote tumor growth by paracrine influences, including vascular endothelial growth factor (VEGF) production and aberrant E-cadherin expression. Finally, a growing body of research over the last decade indicates that epigenetic alterations may silence tumor suppressor genes and that they represent important events in tumor progression.

\section{Signs and Symptoms}

Bladder cancer characteristically causes blood (redness) in the urine. This blood in the urine may be visible to the naked eye (gross/macroscopic haematuria) or detectable only by microscope (microscopic haematuria). Haematuria is the most 
common symptom in bladder cancer. It occurs in approximately $80-90 \%$ of the patients. Other possible symptoms include pain during urination. Frequent urination or feeling the need to urinate without being able to do so. These signs and symptoms are not specific to bladder cancer, and are also caused by noncancerous conditions including; prostate infections, over- active bladder and cystitis.

There are many other causes of haematuria, such as bladder or ureteric stones
i. Infection
ii. Kidney disease
iii. Kidney cancers

\section{Clinical Course}

The clinical course of bladder cancer is marked by a broad spectrum of aggressiveness and risk. Low-grade, superficial bladder cancers have minimal risk of progression to death; however, high-grade non-muscle-invasive cancers frequently progress and muscle-invasive cancers are often lethal. The classic presentation of bladder cancer is painless gross ahematuria, which is seen in approximately $80-90 \%$ of patients. Physical examination results are often unremarkable. Cystoscopy, cytology, and biopsy when necessary are the principal diagnostic tests.

Upon presentation, $55-60 \%$ of patients have low-grade, noninvasive disease, which is usually treated conservatively with transurethral resection of bladder tumor (TURBT) and periodic cystoscopy. Intravesical agents may also be given selectively to decrease the frequency of recurrences. The remaining patients have high-grade disease, of which $50 \%$ is muscle invasive and is typically treated with radical cystectomy or with trimodality therapy (i.e., TURBT followed by concurrent radiation therapy and systemic chemotherapy). Carcinoma in situ (CIS) is managed by TURBT and instillation of chemotherapeutic or immunotherapeutic agents-most commonly, immunotherapy with bacillus Calmette-Guérin (BCG) vaccine-into the bladder via catheter. These intravesical treatments are not effective in the $20 \%$ of patients in whom cancer has invaded the bladder wall muscle; those cases require cystectomy or a combination of radiation therapy and chemotherapy. Bladder cancer has the highest recurrence rate of any malignancy. Although most patients with bladder cancer can be treated with organ-sparing therapy, most experience either recurrence or progression, creating a great need for accurate and diligent surveillance.

\section{Laboratory Diagnosis/Radiology}

Urine studies include the following:

i. Urinalysis with microscopy

ii. Urine culture to rule out infection, if suspected
iii. Voided urinary cytology
iv. Urinary tumor marker testing

\section{Urinary cytology}

Standard noninvasive diagnostic method.

Low sensitivity for low-grade and early stage cancers.

Fluorescence in situ hybridization (FISH) may improve the accuracy of cytology.

\section{Cystoscopy}

i. The primary modality for the diagnosis of bladder carcinoma

ii. Permits biopsy and resection of papillary tumors

\section{Upper urinary tract Imaging}

i. Necessary for the hematuria workup.

ii. American Urologic Association Best Practice Policy recommends computed tomography (CT). scanning of the abdomen and pelvis with contrast, with preinfusion and post infusion phases

iii. Imaging is ideally performed with CT urography, using multidetector CT.

iv. Ultrasonography is commonly used, but it may miss urothelial tumors of the upper tract and small stones.

The diagnostic strategy for patients with negative cystoscopy is as follows:

i. Negative urine cytology and FISH - Routine follow-up

ii. Negative urine cytology, positive FISH - Increased frequency of surveillance

iii. Positive urine cytology, positive or negative FISH Cancer until proven otherwise.

No blood tests are specific for bladder cancer, but a general evaluation is necessary prior to initiating therapy with intravesical bacillus Calmette-Guérin (BCG) vaccine. Laboratory tests include the following:

a. Complete blood count (CBC)

b. Liver function tests

c. Bony fraction of alkaline phosphatase assay (if bone metastasis suspected)

d. Kidney function studies

\section{Treatment of Bladder Cancer}

\section{Superficial Bladder Cancer}

Superficial bladder cancer is bladder cancer has not invaded into the muscle. The extent of disease is based mainly on findings 
during the transurethral resection (TUR). Likewise, the primary treatment for superficial disease is the TUR. Since the cancer is superficial, all of the tumor may be able to be removed by the TUR. Following initial treatment with a TUR procedure a patient will undergo intravesicular chemotherapy.

Intravesicular chemotherapy involves the instillation of chemotherapy directly into the bladder so that any remaining cancer cells can be eradicated. Mitomycin $\mathrm{C}$ is the most commonly used chemotherapy used for this treatment. For some, the treatment of superficial bladder cancer may continue beyond a TUR and intravesicular chemotherapy [24]. Some patients may also receive intravesicular therapy with a drug called Bacillus Calmette-Guerin (BCG) for a minimum of one year. Patients will also be monitored for relapse or recurrence of tumors [25].

Muscle Invading Bladder Cancer Cystectomy, or the surgical removal of the entire bladder, is the standard of care for treating more advanced cancers. A large concern in performing this surgery is how to divert the urine so that the patient can still excrete it. In the past, this was done using an "ileal conduit", where the urine drained through a portion of the small intestine and out through the skin into a bag. More recently, techniques for bladder reconstruction have developed. This allows the ureters to be implanted into the newly created bladder and the urethra to lead out of the new bladder. These techniques may allow the patient to be continent and urinate normally. Often, chemotherapy may be used in addition to surgery, either before or after the surgery.

Use of chemotherapy may prolong survival and decrease risk of cancer recurrence. The combination of cisplatin and gemcitabine, either before or after cystectomy, has been shown to be as effective but less toxic than the old standard regimen "MVAC" (methotrexate, vinblastine, doxorubicin, cisplatin. In patients unable to receive cisplatin chemotherapy, other chemotherapy agents such as 5-FU and Mitomycin C may be used. Sometimes, transurethral resection (TUR), in combination with radiation and chemotherapy may be used to allow the patient to avoid cystectomy. This is referred to as a "bladderpreservation approach" and is also known as tri- modal therapy. The best candidates for this treatment approach are those with cancer limited to just one area of the bladder that is less than $5 \mathrm{~cm}$ in size, with no blockage of the ureter or kidney, and good bladder function (since those with poor bladder function would be better off with a cystectomy). Regimens that have the best results all start with maximum resection of the bladder tumor via TUR, just like with superficial bladder cancers.

The patient then starts a treatment course of radiation with chemotherapy, usually cisplatin, for 4-5 weeks.

The chemotherapy is used as a "radio sensitizer" which means it helps make the cancer cells more sensitive to the radiation. Patients are then re-evaluated by a repeat cystoscopy to determine if the chemotherapy and radiation have caused the tumor completely disappear. If the tumor is no longer present, further chemotherapy and radiation is given for an additional 2-3 weeks. This method has comparable survival rates to cystectomy and has the advantage of allowing the patient to keep his or her bladder. If invasive disease remains after chemotherapy and radiation, the patient may be advised to undergo cystectomy, despite efforts to avoid this. If superficial disease remains after chemotherapy and radiation, either BCG or a cystectomy may be considered.

\section{Cystectomy}

Cystectomy is performed for patients who do not have adequate response to chemotherapy and radiation in order to eliminate the cancer and reduce the risk of death from the cancer. Even when patients do not need to undergo cystectomy, they may experience side effects from radiation and chemotherapy. The most concerning of these are decreased bladder capacity (leading to more frequent urination), bladder spasm, chronic burning or pain with urination, and hematuria from the damage done by the chemotherapy and radiation [26].

\section{Other type of standard treatment includes}
i. $\quad$ Surgery
ii. Radiation therapy
iii. Chemotherapy
iv. Biologic therapy

\section{Surgery}

One of the following types of surgery may be done:

Transurethral resection (TUR) with fulguration: Surgery in which a cystoscope (a thin lighted tube) is inserted into the bladder through the urethra. A tool with a small wire loop on the end is then used to remove the cancer or to burn the tumor away with high-energy electricity. This is known as fulguration [27].

\section{Radical Cystectomy}

Surgery to remove the bladder and any lymph nodes and nearby organs that contain cancer. This surgery may be done when the bladder cancer invades the muscle wall, or when superficial cancer involves a large part of the bladder. In men, the nearby organs that are removed are the prostate and the seminal vesicles. In women, the uterus, the ovaries, and part of the vagina are removed. Sometimes, when the cancer has spread outside the bladder and cannot be completely removed, surgery to remove only the bladder may be done to reduce urinary symptoms caused by the cancer. When the bladder must be removed, the surgeon creates another way for urine to leave the body.

\section{Surgery to Remove part of the bladder}

This surgery may be done for patients who have a low-grade tumor that has invaded the wall of the bladder but is limited to 


\section{Cancer Therapy \& Oncology International Journal}

one area of the bladder. Because only a part of the bladder is removed, patients are able to urinate normally after recovering from this surgery. This is also called segmental cystectomy. Urinary diversion: Surgery to make a new way for the body to store and pass urine. Even if the doctor removes all the cancer that can be seen at the time of the surgery, some patients may be given chemotherapy after surgery to kill any cancer cells that are left. Treatment given after surgery, to lower the risk that the cancer will come back, is called adjuvant therapy.

\section{Radiation Therapy}

Radiation therapy is a cancer treatment that uses highenergy x-rays or other types of radiation to kill cancer cells or keep them from growing. There are two types of radiation therapy:

External radiation therapy uses a machine outside the body to send radiation toward the cancer.

Internal radiation therapy uses a radioactive substance sealed in needles, seeds, wires, or catheters that are placed directly into or near the cancer.

The way the radiation therapy is given depends on the type and stage of the cancer being treated.

External radiation therapy is used to treat bladder cancer.

\section{Chemotherapy}

Chemotherapy is a cancer treatment that uses drugs to stop the growth of cancer cells, either by killing the cells or by stopping them from dividing. When chemotherapy is taken by mouth or injected into a vein or muscle, the drugs enter the bloodstream and can reach cancer cells throughout the body (systemic chemotherapy) [28]. When chemotherapy is placed directly into the cerebrospinal fluid, an organ, or a body cavity such as the abdomen, the drugs mainly affect cancer cells in those areas (regional chemotherapy). For bladder cancer, regional chemotherapy may be intravesical (put into the bladder through a tube inserted into the urethra). The way the chemotherapy is given depends on the type and stage of the cancer being treated. Combination chemotherapy is treatment using more than one anticancer drug [29].

\section{Biologic Therapy}

Biologic therapy is a treatment that uses the patient's immune system to fight cancer. Substances made by the body or made in a laboratory are used to boost, direct, or restore the body's natural defenses against cancer. This type of cancer treatment is also called biotherapy or immunotherapy [30]. Bladder cancer may be treated with an intravesical biologic therapy called BCG (bacillus Calmette- Guérin). The BCG is given in a solution that is placed directly into the bladder using a catheter (thin tube) [31].

\section{Drugs for Treatment of Bladder Tumor}

i. Atezolizumab

ii. Cisplatin

iii. Doxorubicin Hydrochloride

iv. Platinol (Cisplatin)

v. Platinol-AQ (Cisplatin)

vi. Tecentriq (Atezolizumab)

vii. Thiotepa

In summary, there are different treatment methods available for bladder cancer with curative potential. As is true for many other sites of cancer, regimens have been developed that allow for a higher quality of life after the treatment is completed.

\section{Management}

The treatment of non-muscle-invasive bladder cancer ( $\mathrm{Ta}$, T1, carcinoma in situ [CIS]) begins with transurethral resection of bladder tumor (TURBT). Subsequent treatment is as follows:

i. Small-volume, low-grade Ta bladder cancer - An immediate single, postoperative dose of intravesical chemotherapy

ii. High-risk Ta, T1, and CIS urothelial carcinoma Intravesical BCG vaccine

iii. Persistent or recurrent high-risk disease - Repeat resection prior to additional intravesical therapy (eg, interferon alfa or gamma); consider cystectomy for high-risk disease

iv. The treatment of muscle-invasive bladder cancer is as follows:

v. Radical cystoprostatectomy in men

vi. Anterior pelvic exenteration in women

vii. Bilateral pelvic lymphadenectomy (PLND), standard or extended

viii. Creation of a urinary diversion

ix. Neoadjuvant chemotherapy - May improve cancerspecific survival

Alternatively, a bladder-sparing approach of TURBT followed by concurrent radiation therapy and systemic chemotherapy (trimodality therapy) may be used.

Chemotherapeutic regimens for metastatic bladder cancer include the following: 


\section{Cancer Therapy \& Oncology International Journal}

a. Methotrexate, vinblastine, doxorubicin (Adriamycin), and cisplatin (MVAC)

b. Gemcitabine and cisplatin (GC) [31].

\section{Prevention and control}

There is no sure way to prevent bladder cancer, but some risk factors have been identified, which may increase your risk of developing the condition, but there are things one can do that could lower the risk.

\section{Stopping Smoking}

If one smokes, giving up is the best way to reduce the person's risk of developing bladder cancer and preventing it from recurring.

\section{Workplace Safety}

One's risk of bladder cancer could be increased if your job involves exposure to certain chemicals. Occupations linked to an increased risk of bladder cancer are manufacturing jobs involving: rubber, dyes, textiles, plastic, leather tanning, and diesel fumes. Nowadays, there is rigorous safety protocols in place designed to minimize one's risk of exposure, and chemicals known to increase the risk of bladder cancer have been banned.

\section{Diet}

There's some evidence to suggest that a diet high in fruit and vegetables and low in fat can help to prevent bladder cancer. Even though this evidence is limited, it's a good idea to follow this type of healthy diet, as it can help to prevent other types of cancer, such as bowel cancer, as well as serious health conditions, including high blood pressure (hypertension), stroke and heart disease. A low-fat, high-fiber diet is recommended, including plenty of fresh fruit and vegetables (five portions a day) and whole grains. Limit the amount of salt you eat to no more than $6 \mathrm{~g}(0.2 \mathrm{oz})$ a day (one teaspoon) because too much can increase your blood pressure. one should try to avoid foods that contain saturated fat, as it can increase your cholesterol levels. Foods high in saturated fat include: meat pies sausages and fatty cuts of meat, butter ghee (a type of butter often used in Indian cooking), lard, cream, cakes and biscuits, foods that contain coconut or palm oil.

\section{Conclusion}

The bladder is a hollow organ in the pelvis with flexible, muscular walls. Its main function is to store urine before it leaves the body. Urine is made by the kidneys and is then carried to the bladder through tubes called ureters. The bladder is an organ located in the lower abdominal area near the pelvic bones that acts as a reservoir for urine. All small cell carcinomas of the urinary system identified so far have been located in the urinary bladder, most commonly in the dome and vesical lateral wall. Bladder cancer begins when cells in the urinary bladder start to grow uncontrollably. As more cancer cells develop, they can form a tumor and spread to other areas of the body. Normally, cells in the body will grow and divide to replace old or damaged cells in the body. This growth is highly regulated, and once enough cells are produced to replace the old ones, normal cells stop dividing. Tumors occur when there is an error in this regulation and cells continue to grow in an uncontrolled way. Tumors can either be benign or malignant.

\section{References}

1. Keimling M, Behrens G, Schmid D, Jochem C, Leitzmann MF (2014) The association between physical activity and bladder cancer: systematic review and meta- analysis. Br J Cancer 110(7): 1862-1870.

2. Dietrich K, Demidenko E, Schned A, Zens MS, Heaney J, et al. (2011) Parity, early menopause and the incidence of bladder cancer in women: A case-control study and meta-analysis. Eur J Cancer 47 (4): 592-609.

3. Davis-Dao CA, Henderson, KD, Sullivan-Halley J, Ma H, West D, et al. (2011) Lower risk in parous women suggests that hormonal factors are important in bladder cancer etiology. Cancer Epidemiology Biomarkers Prev 20(6): 1156-1170.

4. Amaral AF, Cantor KP, Silverman DT, Malats N (2010) Selenium and bladder cancer risk: a meta- analysis. Cancer Epidemiology Biomarkers Prev 19(9): 2407-2715.

5. Daugherty SE, Lacey JV, Pfeiffer RM (2013) Reproductive factors and menopausal hormone therapy and bladder cancer risk in the NIHAARP Diet and Health Study. Int J Cancer 133(2): 462- 472.

6. Liang S, Lv G, Chen W, Jiang J, Wang J (2014) Citrus fruit intake and bladder cancer risk: a meta-analysis of observational studies. Int J Food Sci Nutr 65(7): 893-898.

7. Yao B, Yan Y, Ye X, Fang H, Xu H, et al. (2014) Intake of fruit and vegetables and risk of bladder cancer: a dose-response meta-analysis of observational studies. Cancer Causes Control 25(12): 1645-1658.

8. Brown T, Slack R, Rushton L (2012) Occupational cancer in Britain Urinary tract cancers: bladder and kidney. Br J Cancer 19(1): 76-84.

9. Tsuji JS, Alexander DD, Perez V, Mink PJ (2014) Arsenic exposure and bladder cancer: quantitative assessment of studies in human populations to detect risks at low doses. 20(3): 17-30.

10. Guha N, Steen land NK, Merletti F (2010) Bladder cancer risk in painters: a meta- analysis. Occupational and Environmental Medical journal 67(8): 568-573.

11. Rota M, Bosetti C, Boccia S, Boffetta P, La Vecchia C (2014) Occupational exposures to polycyclic aromatic hydrocarbons and respiratory and urinary tract cancers. Arch Toxicol 88(8): 1479-1490.

12. Manju L, George PS, Mathew A (2009) Urinary bladder cancer risk among motor vehicle drivers: a meta-analysis of the evidence, 19772008. Asian Pac J Cancer Prev 10(2): 287-294.

13. Takkouche B, Regueira-Méndez C, Montes M (2009) A Risk of cancer among tobacco smoking and bladder cancer risk. Cancer Causes and Control 13(1): 83-90.

14. Zeegers MP, Tan FE, Dorant E, Brandt PA (2000) The impact of characteristics of cigarette smoking on urinary tract cancer risk: a meta-analysis of epidemiologic studies. Cancer 89(3): 630-639.

15. Osch FHM, Jochems SHJ, Schooten FV, Bryan RT, Zeegers MP (2016) Quantified relations between exposure to tobacco smoking and bladder cancer risk: a meta-analysis of 89 observational studies. Int J Epidemiol 45(3): 857-870.

16. Reulen RC, Zeegers MP, Buntinx F, Brinkman M, Zeegers MP, et al. (2008) A meta- analysis on the association between bladder cancer and occupation. Scand J Urol Nephrol Suppl 218: 64-78. 


\section{Cancer Therapy \& Oncology International Journal}

17. Al-Zalabani K, Abdulmohsen H, Stewart Kelly FJ, Wesselius A, Annemie MJ, et al. (2016) Modifiable risk factors for the prevention of bladder cancer: a systematic review of meta-analyses. European Journal of Epidemiology 31(9): 1-41.

18. Valtin H (2002) Cancer reduction. American Journal of Physiology 3(2): 120-135.

19. Walid MS, Heaton RL (2008) Can posthysterectomy cystoscopy be utilized as a screening test for bladder cancer. German Medical Science 6: $56-71$.

20. Lotan Y, Roehrborn CG (2003) Sensitivity and specificity of commonly available bladder tumor markers 7(11): 598-600.

21. Chou R, Dana T (2010) Screening adults for bladder cancer: a review of the evidence for the U.S. preventive services task force. Ann Intern Med 153(7): 461-468.

22. Sherif A, Holmberg L, Rintala E, Mestad O, Nilsson J, et al. (2004) Neoadjuvant cisplatinum based combination chemotherapy in patients with invasive bladder cancer: a combined analysis of two Nordic studies. Eur Urol 45(3): 297-300.

23. Grossman, HB, Natale RB, Tangen CM, Speights VO, Vogelzang NJ, et al. (2003) Neoadjuvant chemotherapy plus cystectomy compared with cystectomy alone for locally advanced bladder cancer. New England Journal of Medicine. 349(9): 859-866.
24. Halachmi S, Moskovitz B, Maffezzini M, Conti G, Verweij F, et al (2009) Intravesical mitomycin $C$ combined with hyperthermia for patients with T1G3 transitional cell carcinoma of the bladder. Urologic Oncology. 29(3): 259-264.

25. Nativ O, Witjes JA, Hendricksen K (2009) Combined thermochemotherapy for recurrent bladder cancer after bacillus CalmetteGuerin. J Urol 182(4): 1313-1337.

26. Di Stasi SM, Riedl C (2009) Updates in intravesical electromotive drug administration of mitomycin-C. World J Urol 27(3): 325-330.

27. Witjes JA, Hendricksen K (2008) Intravesical pharmacotherapy for non-muscle-invasive. 51(3): 45-52.

28. Lamm DL, Blumenstein BA, Crawford E, David M, James E, et al. (1991) A Randomized Trial of Intravesical Doxorubicin and Immunotherapy with Bacille Calmette-Guérin for Transitional-Cell Carcinoma of the Bladder. New England Journal of Medicine 325(17): 1205-1209.

29. Witjes JA (2006) Management of BCG failures in superficial bladder cancer: a review. Eur Urol 49(5): 790-797.

30. Alexandroff AB, Jackson AM, O’Donnell MA, James K (1999) BCG immunotherapy of bladder cancer: 20 years on. Lancet 353(5): 169194.

31. Michelle Roberts (2013) Urine odor test for bladder cancer. BBC News London, UK, p. 13

Your next submission with Juniper Publishers will reach you the below assets

- Quality Editorial service

- Swift Peer Review

- Reprints availability

- E-prints Service

- Manuscript Podcast for convenient understanding

- Global attainment for your research

- Manuscript accessibility in different formats

( Pdf, E-pub, Full Text, Audio)

- Unceasing customer service

Track the below URL for one-step submission https://juniperpublishers.com/online-submission.php 